\title{
HYBRIDS IN TELECOMMUNICATIONS
}

\author{
D. ROGGIA \\ Telettra S.p.A., 20059 Vimercate (MI), Italy
}

\begin{abstract}
The telecommunication industry has greatly contributed to the diffusion of hybrid technology in all electronic fields. The approach, followed by almost every telecommunication company, in order to optimize the implementation of hybrid circuits, is to use both thin and thick film technologies. After a short review of the characteristics of the telecommunication market, which affect hybrid technology, the adaptive and simple but powerful approach of Telettra will be presented.
\end{abstract}

\section{THE CHARACTERISTICS OF THE TELECOMMUNICATION MARKET}

In this paper telecommunication is considered to be telephony, i.e. the service which allows people to communicate each other, whenever they want to and however distant they are. This is accomplished through a world-wide network whose structure may be roughly divided into three broad areas:
1) customer,
2) switching and
3) transmission.

All the signals generated on the customer's equipment are taken to a switching office and then routed to the selected receiving station. For long distance delivery, signals arrive from many switching offices at a transmission line terminal. To conserve bandwidth, these signals are processed and grouped through a multiplexing technique and then transmitted.

Transmission occurs via coaxial cable, microwave radio relay, under water cable, or satellite.

The structure of this network is such as to require a very large number of different equipments organized on many hierarchial levels, all of them, for various reasons, using hybrid circuits. There are equipments dedicated to each subscriber and others are shared, either in turn or simultaneously by as many as 10,000 customers. As far as hybrid circuits are concerned, this results in a high "production dispersion," i.e. a wide variety of circuits to be manufactured on very different yearly volumes, with the risk of lessening the production efficiency and capability.

Figure 1 shows a typical distribution of the number of circuits as a function of the required annual production.

The right side of the curve could represent as much as $80 \%$ by volume, but perhaps less than $50 \%$ by value.

The high capital investment required for new telecommunication systems and the vital role they play as a social service, require all equipment to have high quality and reliability; a life of at least 25 years is quite usual, with a target for some new equipment of a Mean Time to Failure greater than 100 years. Another important aspect of this market is its steady growth-rate, not only in terms of number of equipments, but also as types of services. This continuously requires new, more sophisticated equipment, at the lowest cost in order to encourage the diffusion of the service. It is clear why many technological breakthroughs have originated in the telecommunication industry.

These peculiarities are just a few of the complex characteristics of telecommunications, but they are very important to the hybrids manufacturer because

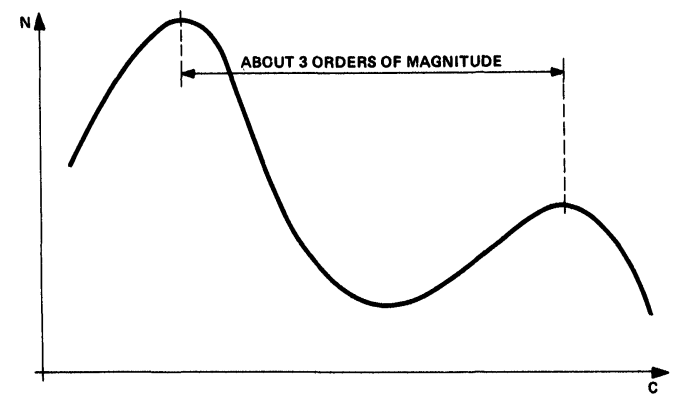

FIGURE 1 Products distribution; number of circuits, N, vs. the required annual production, $\mathrm{C}$. 


\begin{tabular}{ll}
\hline & - in-house capability \\
Production Dispersion & - processes as standard as possible, \\
Low Cost & but still flexible \\
& "batch" process approach \\
\hline & - well established processes \\
& - strict controls of materials \\
Quality and Reliability & and processes \\
\hline Innovation & - competent R and D staff \\
& - keep track of new technologies \\
\hline
\end{tabular}

their requirements are often conflicting. Table I summarizes these requirements and how a telecommunication company can comply with them.

The need of an in-house capability must be emphasized. It could be said that it is obvious that a telecommunication company must have a laboratory facility, for designing new circuits, setting down specifications, etc. However as the main purpose of the company is to produce and sell equipments and systems, it would be more economical to buy hybrids outside, unless the volumes were such to saturate a production line. This holds true for semiconductors or discrete passive components, but in the case of the hybrids we must keep in mind that they are custom products, whose availability is essential for producing the equipment. One external supplier cannot be relied on. On the other hand it would be extremely expensive to have two external suppliers on some hundred types of circuits, some of them requiring just a few hundred pieces per year.

The only possibility is to produce circuits inside the company and perhaps to have an external supplier as second source for the highest volume circuits. But being "captive" manufacturers puts us in a difficult situation: our costs are continuously compared with the best outside prices, allowing only a small corrective factor to take into account our strategic function.

\section{TELETTRA'S APPROACH}

Let us now examine how we realized the objectives shown in Table I in Telettra. When we decided to set up a hybrid technology in 1966 we aimed at the following characteristics:-

1) high performances
2) applicable both at low and high frequencies

3) requiring progressive capital investment for best tradeoff volume/cost

4) appreciable content of original know-how.

Then the choice had been for tantalum thin film.

By 1974 we had put in production a broad spectrum of circuits, intended for both FDM and PCM Multiplexes, line systems, radio relays, electronic switching exchanges, remote supervisory, control equipments.

Figures 2 and 3 show two examples of application, intended respectively for FDM and Radio - repeater equipments.

Thus we used thin film circuits where either electrical performances, quality or volume requirements justified their use.

At the same time we were saturating the line capability, though still having demands for low cost applications.

At that point, as thick film materials and processing techniques (particularly laser trimming) had developed, we introduced the thick film technology to complement thin film. All the medium and large-size telecommunication companies today have both technologies; but the criteria for choosing the one or the other is usually the frequency range or the production volume. Typical of companies which started using thick film first is that they have been forced, or tempted, to obtain from thick film almost the same performances inherent in thin film, perhaps introducing some critical steps in the process such that, even when completely developed production is costly.

Our criterion has been to develop the simplest and cheapest thick film process, compatible with reliability requirements, in order to get real cost effectiveness, whatever the frequency and the production volume. 


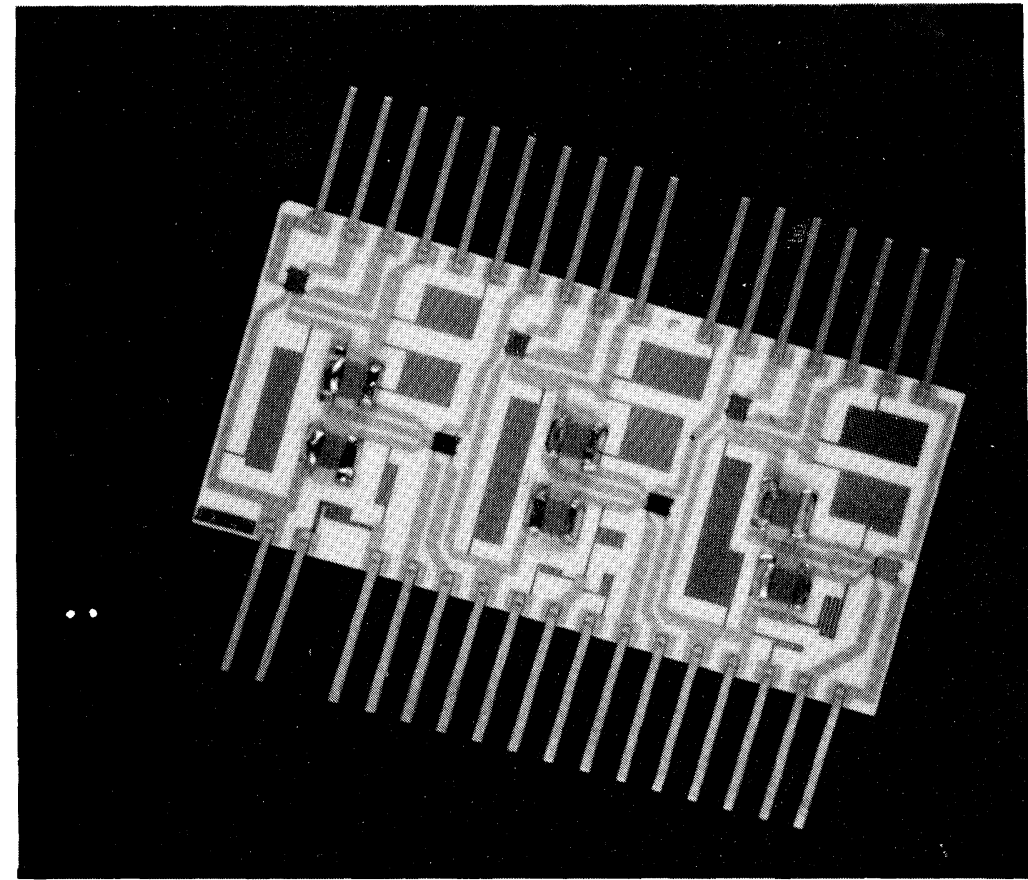

FIGURE 2 Thin film pass band filter; with chip capacitors and beam-leaded op-amps.

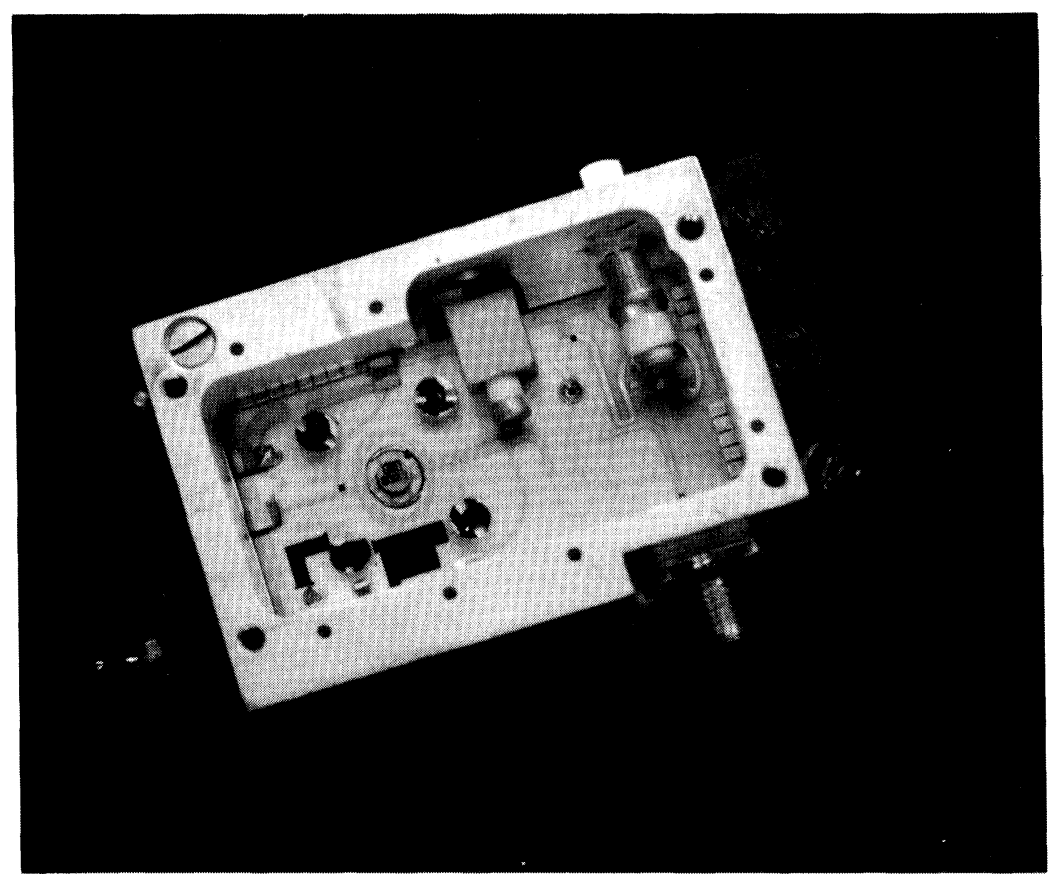

FIGURE $3 \quad 2.3 \mathrm{GHz}$ oscillator, for a phase-locked injection repeater. 
TABLE II

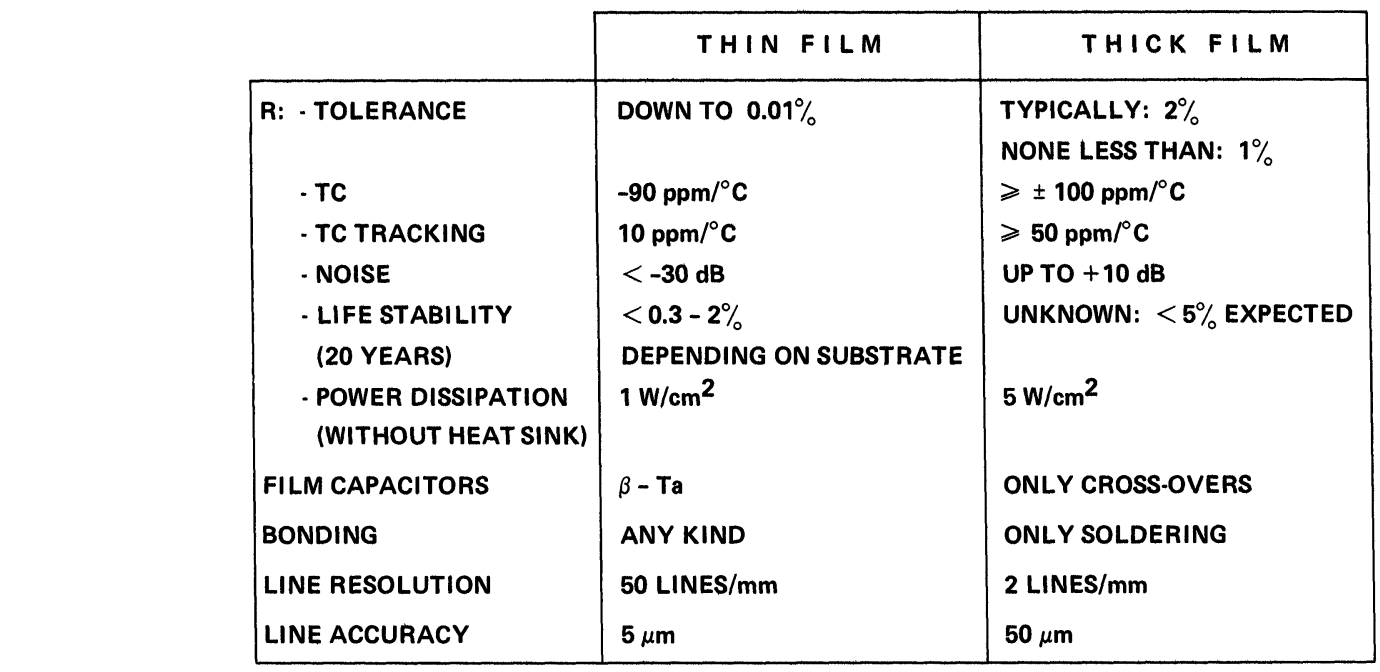

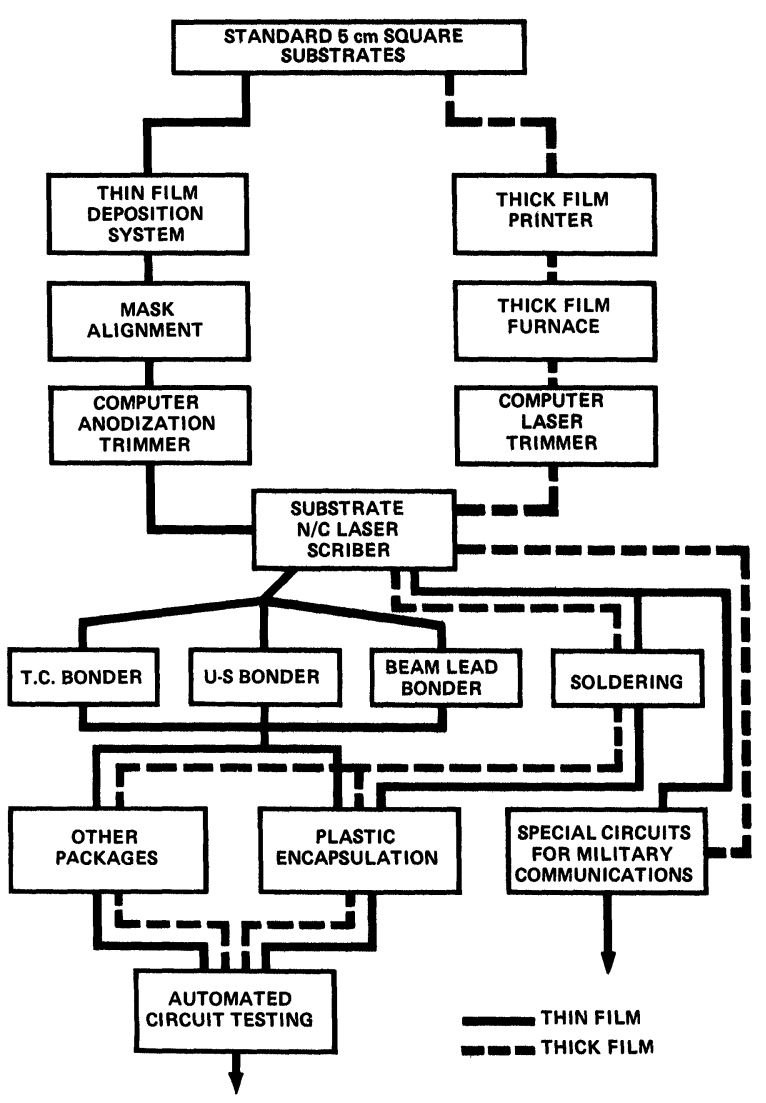

FIGURE 4 Hybrid processes flow diagram.

This means that we use the one or the other, only on the basis of the electrical performances required. Table II shows the specifications of our processes.
For the sake of simplicity, "thin film" refers to $\mathrm{Ta}_{2} \mathrm{~N}$ resistors only; with minor changes in the process we can also obtain tantalum-oxy-nitride resistors, with still improved performances. Moreover, the figures given do not always represent the very best achievable, for thin or thick-films, but are the result of the design rules we use in order to guarantee high production yield and reliability.

To further lower the costs, the final steps go through the same automatic equipment already set up for some thin film products. Figure 4 shows how production is organized for this purpose, and Figure 5 shows an application where both thin and thick film are used, according to the above mentioned criteria, attaining the minimum production cost.

\section{FUTURE TRENDS}

The hybrid technology, as now used for telecommunications, will not go through dramatic changes in the near future. A lot of work has still to be done, mainly concerning yield increase, automation, and use of non-precious metals. There may be a decrease in the use of active hybrid circuits, particularly in the low frequency range, due to the permanent scarcity of cheap, reliable IC-package, suitable for hybrid integration.

In the medium-long term, we must look carefully at some new devices, which taking advantages of some techniques commonly used for the hybrids, seem to be their natural but not immediate extension.

Examples are the surface acoustic wave devices and the integrated optical circuits. They are expected 


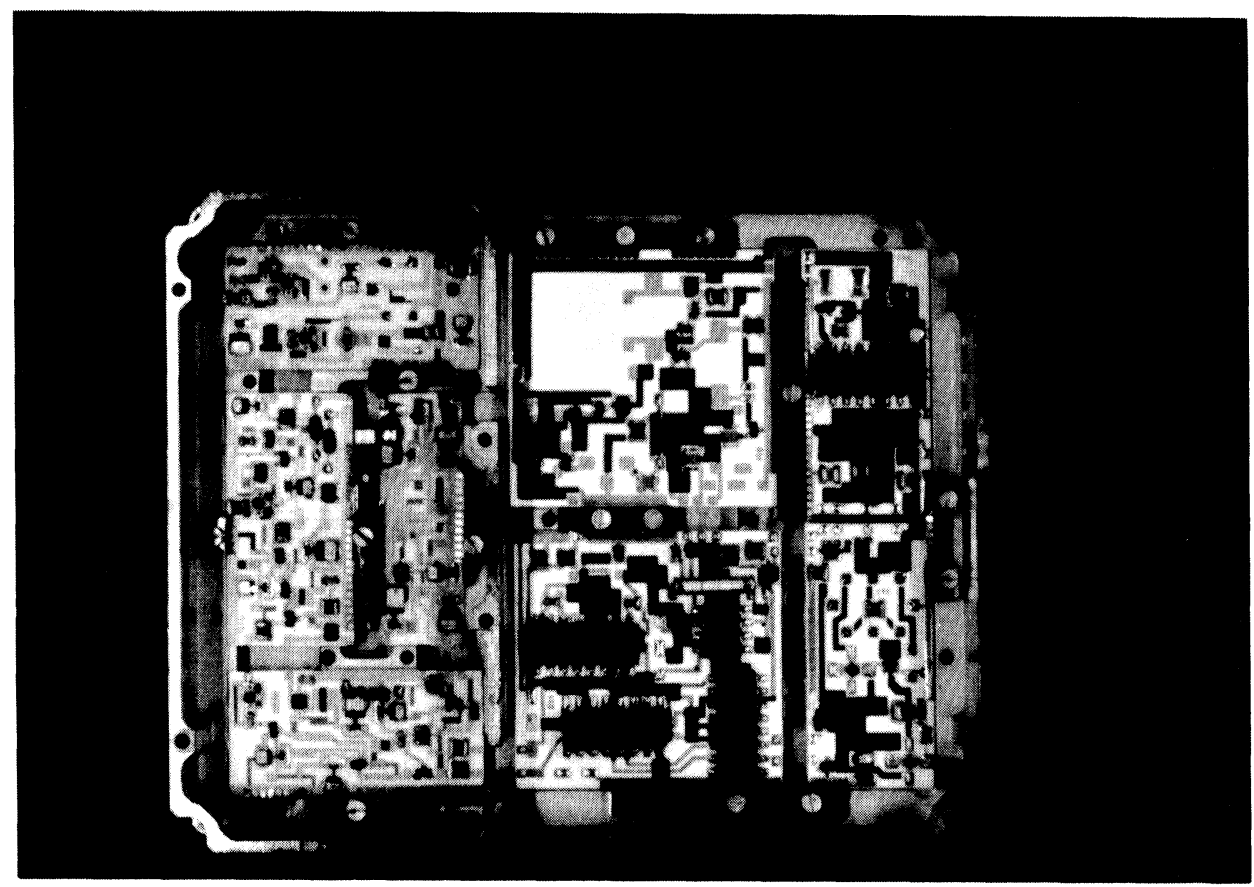

FIGURE $5140 \mathrm{Mbit} / \mathrm{s}$ line regenerator.

to play an important role in future integrated communication networks. The former will permit functions which have been extremely difficult to perform in the past, while the latter will allow, efficiently and economically, new wideband services. $\mathrm{R}$ and $\mathrm{D}$ efforts, all through the world, show steady advances. Many problems, however, remain to be solved, such as basic materials, processing techniques, packaging, reliability and economy.

When developed these new technologies will not replace hybrid circuits, but together they will contribute to open up and make economical new communications services. 

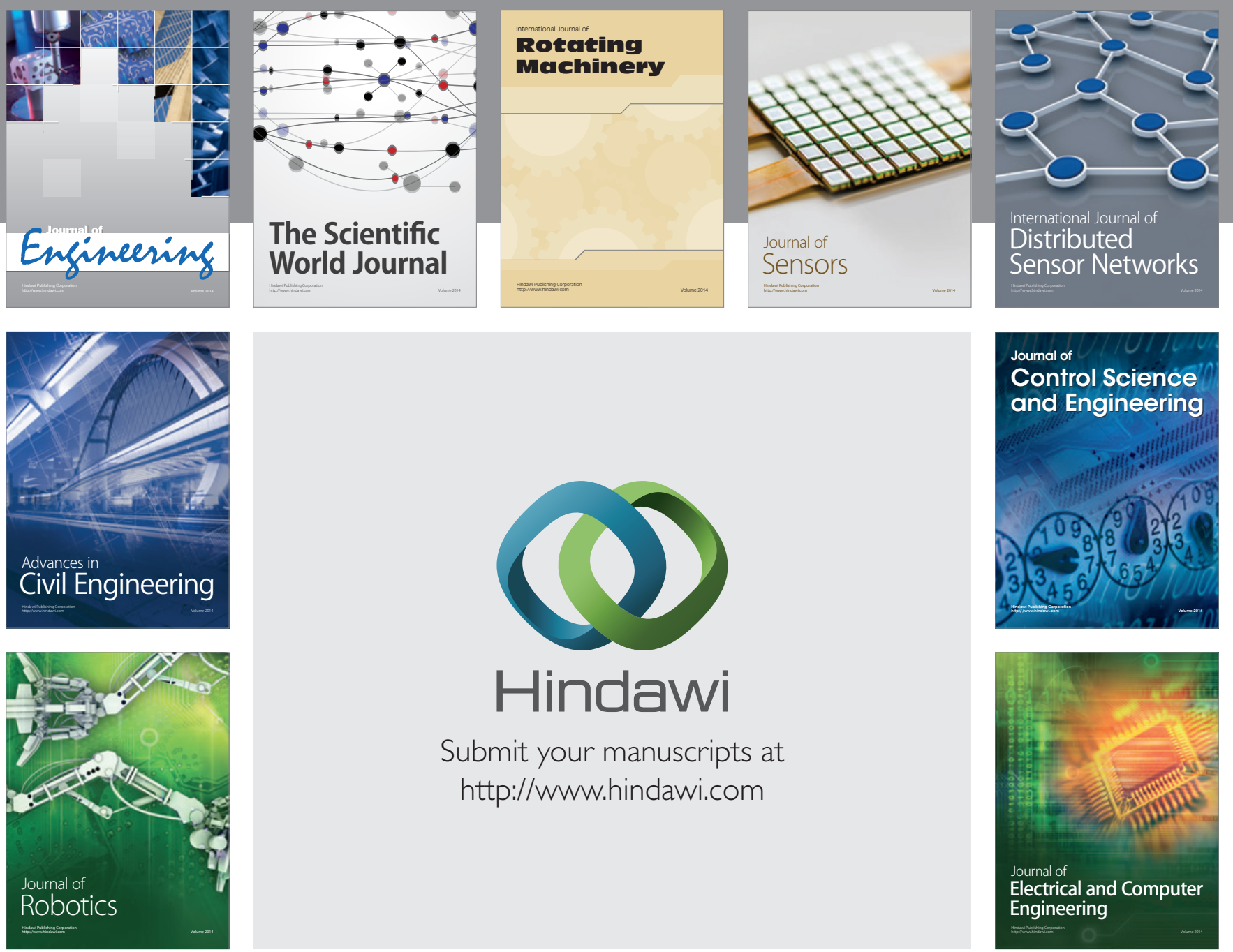

Submit your manuscripts at

http://www.hindawi.com
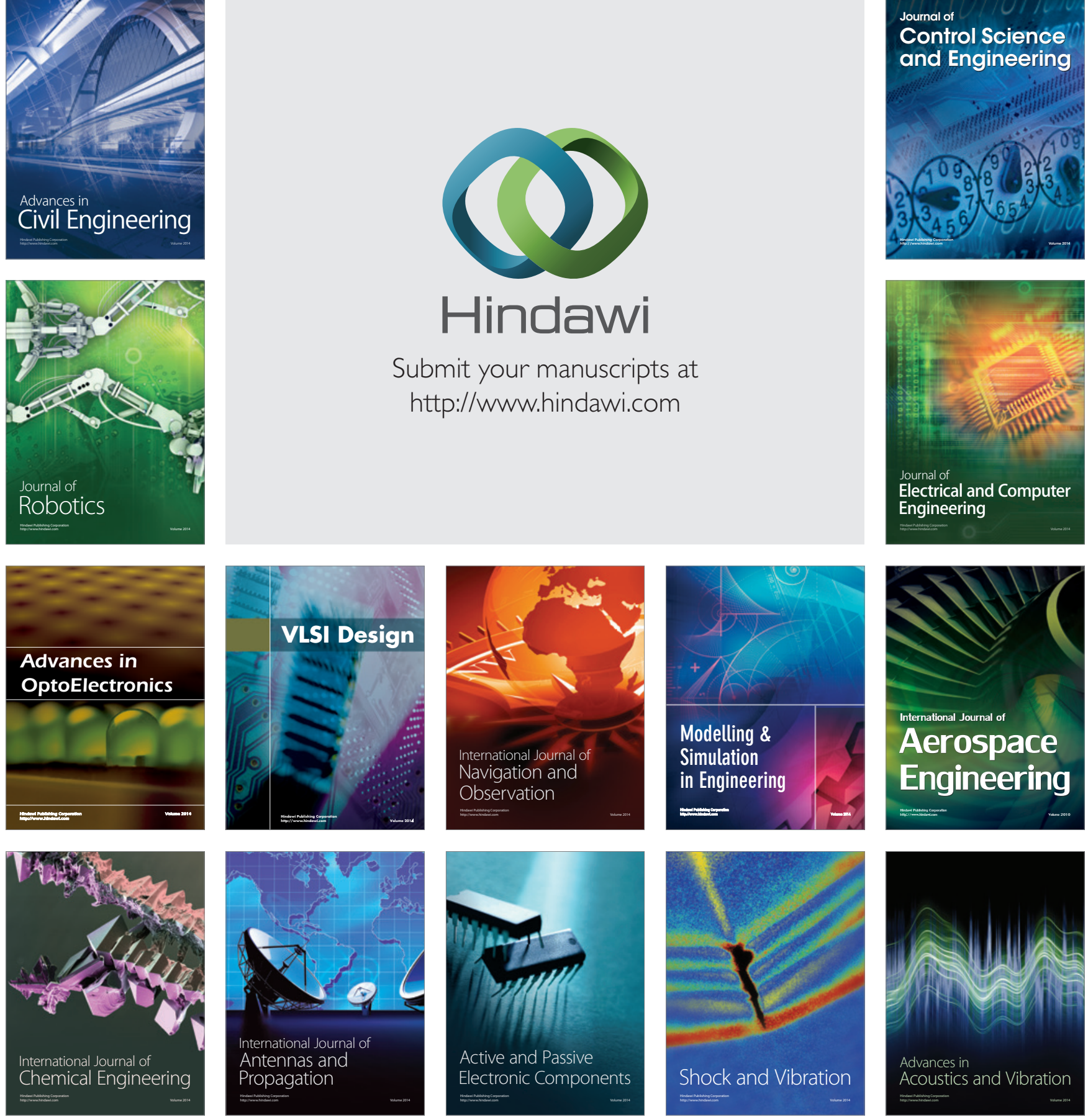